\title{
Option Pricing under the Subordinated Market Models
}

\author{
Longjin Lv $\mathbb{D}$, Changjuan Zheng $\mathbb{D}$, and Luna Wang $\mathbb{1}$ \\ School of Finance and Information, Ningbo University of Finance and Economics, Ningbo 315000, China \\ Correspondence should be addressed to Longjin Lv; magic316@163.com
}

Received 10 July 2021; Revised 24 November 2021; Accepted 28 December 2021; Published 15 January 2022

Academic Editor: Benjamin Miranda Tabak

Copyright (c) 2022 Longjin Lv et al. This is an open access article distributed under the Creative Commons Attribution License, which permits unrestricted use, distribution, and reproduction in any medium, provided the original work is properly cited.

\begin{abstract}
This paper aims to study option pricing problem under the subordinated Brownian motion. Firstly, we prove that the subordinated Brownian motion controlled by the fractional diffusion equation has many financial properties, such as self-similarity, leptokurtic, and long memory, which indicate that the fractional calculus can describe the financial data well. Then, we investigate the option pricing under the assumption that the stock price is driven by the subordinated Brownian motion. The closed-form pricing formula for European options is derived. In the comparison with the classic Black-Sholes model, we find the option prices become higher, and the "volatility smiles" phenomenon happens in the proposed model. Finally, an empirical analysis is performed to show the validity of these results.
\end{abstract}

\section{Introduction}

Brownian motion and normal distribution have been widely used in the Black-Scholes option pricing framework to the return of assets. The classical Black-Scholes model is based on the diffusion process called geometric Brownian motion $(\mathrm{GBM})[1,2]$.

$$
d S_{t}=\mu S_{t} d t+\sigma S_{t} d B(t)
$$

where $\mu$ and $\sigma$ are constants and $B(\tau)$ is the standard Brownian motion.

However, on the one hand, in the last few years, the empirical studies have shown that many characteristic properties of markets cannot be captured by the BS model [3], such as heavy tailed, long-range correlations, and lack of scale invariance and periods of constant values [4].

On the other hand, in a complete financial market without transaction costs, the Black-Scholes no-arbitrage argument provides a hedging portfolio that replicates the option. However, the Black-Scholes hedging portfolio requires continuous trading, and therefore, in a market with propositional transaction costs, it is expensive. Leland [5] first examined option replication in the presence of transaction costs (TC) in a discrete time setting and posed a modified replicating strategy, which depends upon the level of transactions costs and upon the revision interval, as well as upon the option to be replicated and the environment. Since then, a lot of authors study this problem, all in a discrete time setting $[6,7]$.

Notably, in a variety of cases, GBM has failed to reproduce the properties of real asset prices. For instance, by definition, GBM is not able to adequately reproduce fattailed distributions of various characteristics observed in reality. In the paper [8], Magdziarz applied the subdiffusive mechanism of trapping events to describe properly financial data exhibiting periods of constant values and introduced the subdiffusive geometric Brownian motion (SGBM) $S_{t}=$ $X\left(S_{\alpha}(t)\right)$ as the model of asset prices exhibiting subdiffusive dynamics. Here, the parent process $X(\tau)$ is the GBM given by equation (1), and $S_{\alpha}(t)$ is the inverse time $\alpha$-stable subordinator with the parameter $\alpha \in(0,1)$, which can be expressed as

$$
\begin{aligned}
d X(\tau) & =\mu X(\tau) d \tau+\sigma X(\tau) d B(\tau), \\
S_{\alpha}(t) & =\inf \left\{\tau>0: U_{\alpha}(\tau)>t\right\},
\end{aligned}
$$

where $U_{\alpha}(\tau)$ is a strictly increasing $\alpha$-stable Levy process with Laplace transform given by $E\left(e^{-k U_{\alpha}(\tau)}\right)=e^{-\tau k^{\alpha}}, 0<\alpha<1$, and $S_{\alpha}(t)$ is independent of $B(\tau)$. Magdziarz showed that the considered model is arbitrage-free but incomplete since the equivalent martingale 
measure is not unique. But, under a special equivalent martingale measure, they obtained the corresponding subdiffusive BS formula for the fair prices of European options. In $[9,10]$, Magdziarz extend the $\alpha$-stable subordinator to a more general Levy subordinator, and the corresponding BS formula is also obtained. This subdiffusive approach assumes anomalous price dynamics. Concretely, the observation that the distribution of log returns is fat-tailed can be attributed to prolonged periods, in which the price of the asset exhibits approximately constant extreme values. These constant periods can be considered to be trapping of particles, as is done in physical systems that manifest anomalous diffusion (subdiffusion) [11-13]. The correlated continuous time random walk is also employed to describe the stock price, and the option pricing formula is obtained in [14]. In $[15,16]$, Aguilar showed that the price of an European call option, whose underlying asset price is driven by the spacetime fractional diffusion, can be expressed in terms of rapidly convergent double-series. The stock is supposed to be driven by Levy processes in [17-19], but the variance of this process does not exist.

Since, in financial market, the expected return is linearly correlative to time and the variance exists. So, in this paper, we suppose that the underlying of the option contract is driven by a subordinated Brownian motion, i.e., the price of underlying $S_{t}$ follows the stochastic differential equation:

$$
d S_{t}=\mu S_{t} d t+\sigma S_{t} d B\left(S_{\alpha}(t)\right)
$$

Here, we suppose the underling pays no dividends.

The paper is organized as follows. In Section 2, we study the self-similarity, leptokurtic, and long memory features of the subordinated geometric Brownian motion, which imply that the supposed model is suitable to describe the asset price. In Section 3, we apply this model to option pricing problem. The closed form representation of the option pricing formula is derived. In Section 4, we present our conclusions.

\section{The Subordinated Brownian Motion}

In this section, we will study the properties of the subordinated geometric Brownian motion (4) to justify our hypothesis.

Firstly, since the strictly increasing $\alpha$-stable Levy process $U_{\alpha}(\tau)$ is $1 / a$ self-similar, it is not difficult to prove that the inverse time $S_{\alpha}(t)$ is $\alpha$ self-similar by its definition (3). The independence between $S_{\alpha}(t)$ and $B(\tau)$ leads $B\left(S_{\alpha}(t)\right)$ to be $a / 2$ self-similar. The self-similarity is a quite important property in financial market [20].

Secondly, the probability density function $p(x, t)$ of volatility term $B\left(S_{\alpha}(t)\right)$ has been proved to satisfy the following fractional diffusion equation [21]:

$$
\frac{\partial^{\alpha}}{\partial t^{\alpha}} p(x, t)=\frac{1}{2} \frac{\partial^{2}}{\partial x^{2}} p(x, t)
$$

with initial condition $p(x, 0)=\delta(x)$. Here, the fractional derivative is also the Caputo type. This equation was first derived from the continuous-time random walk scheme with heavy-tailed waiting-times [22]. It was proved to be subdiffusive $(0<\alpha<1)$. Subdiffusion is a well-known and established phenomenon in statistical physics. The usual model of subdiffusion in physics is developed in terms of FFPE (fractional Fokker-Planck equations). It provides a useful way for the description of transport dynamics in complex systems [21]. The empirical data indicate the subdiffusive nature exits in the short rate dynamics [23].

In [22], the authors studied the asymptotic behavior of the solution of the fractional diffusion equation. They found $\log p(x, t) \sim C \xi^{u}$, where $\xi \equiv x / t^{a / 2} \gg 1, \quad u=1 /(1-a / 2)$, which means the solution follows a stretched Gaussian distribution. In other words, the solution $p(x, t)$ for equation (4) has heavy tail and high peak, in contrast to the normal distribution. In [24], we can find the figure of the solution, and the simulated results can be found in [25]. The heavy tail and high peak phenomenon (always called leptokurtic feature) is quite common in the financial historical data $[4,26]$, such as the return risk of the assets. Fractional calculus has become a useful tool to fit the financial data [27-30].

From the definition of $S_{\alpha}(t)$, we know that, for every jump of $U_{\alpha}(\tau)$, there is a corresponding flat period of its inverse $S_{\alpha}(t)$. These heavy-tailed flat periods of $S_{\alpha}(t)$ represent long waiting-times in which the subdiffusive particle gets immobilized in the trap (see Figure 1). When $\alpha \nearrow 1, S_{\alpha}(t)$ reduces to the "objective time" $t$.

Thirdly, as we know, the PDF of $B\left(S_{\alpha}(t)\right)$ follows the fractional diffusion equation, where the Caputo fractional operator is given as

$$
{ }_{0} D_{t}^{a} f(t)=\frac{1}{\Gamma(1-\alpha)} \int_{0}^{t}(t-s)^{-a} \frac{\mathrm{d} f(s)}{\mathrm{d} s} \mathrm{~d} s, \quad 0<\alpha<1 .
$$

From the definition of Caputo fractional derivative, we know the fractional derivative at time $t$ is not defined locally, and it relies on the total effects of the commonly used firstorder integer derivative on the interval $[0, t]$. So, it can be used to describe the variation of a system in which the instantaneous change rate depends on the past state, which is called memory effect. Because of the kernel function $\phi(t)=(t-s)^{-\alpha} / \Gamma(1-\alpha)$, the process $B\left(S_{\alpha}(t)\right)$ turns out to be a long-memory processes, namely, non-Markovian processes characterized by a memory function $\phi(t)$ exhibiting a power-law time decay [28]. However, there is substantial evidence that long memory processes describe rather well-financial data such as forward premiums, interest rate differentials, and inflation rates. Perhaps, the most dramatic empirical success of long memory processes has been in recent work on modeling the volatility of asset prices and power transformations of returns[31].

In addition, let $r(\Delta t)$ be the return of the stock $S_{t}$ in the period of time $\Delta t$, defined by $r(\Delta t)=S_{t+\Delta t}-S_{t} / S_{t}$. In discrete time, equation (3) turns to

$$
S_{t+\Delta t}-S_{t}=\mu S_{t} \Delta t+\sigma S_{t}\left(B\left(S_{\alpha}(t+\Delta t)\right)-B\left(S_{\alpha}(t)\right)\right) .
$$

Then, the expected return of the stock $E[r(\Delta t)]=\mu \Delta t$, which is linearly correlative to time. But the expected return turns to $E[r(\Delta t)]=\mu(\Delta t)^{\alpha} / \Gamma(\alpha+1)$ in [8]. 


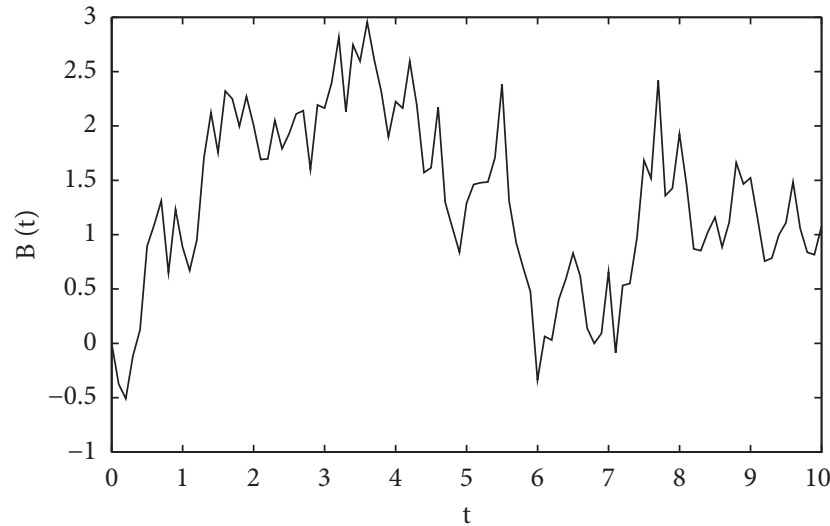

(a)

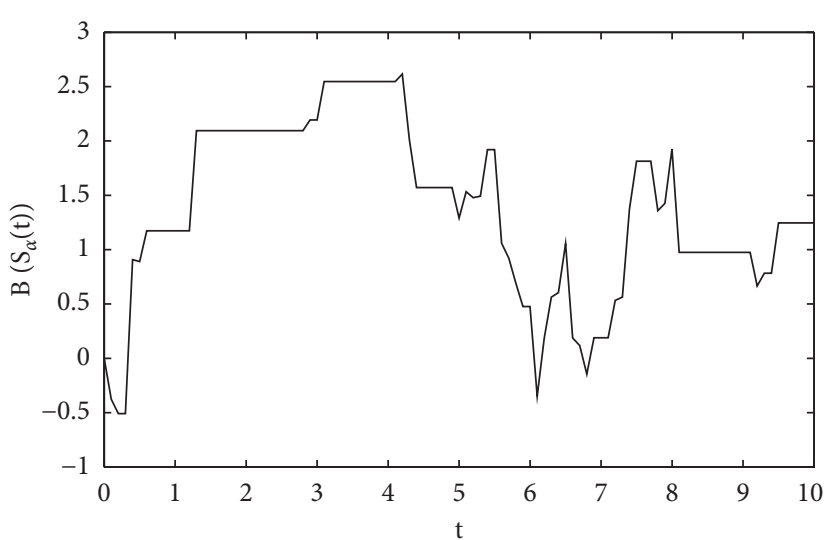

(b)

Figure 1: Comparison of trajectory of the standard Brownian motion $B(t)$ (a) and the subordinated Brownian motion $B\left(S_{\alpha}(t)\right)(\mathrm{b})$ with $\alpha=0.7$.

The scaling role is also satisfied by $B\left(S_{\alpha}(t)\right)$,

$$
\begin{aligned}
\mathbb{E}\left[\left|B\left(S_{\alpha}(t)\right)\right|^{q}\right] & =\mathbb{E}\left[\left|S_{\alpha}(t)\right|^{q / 2}\right] \mathbb{E}\left[|B(1)|^{q}\right] \\
& =\frac{\Gamma(q / 2+1)}{\Gamma(q \alpha / 2+1)} \mathbb{E}\left[|B(1)|^{q}\right] \times t^{q a / 2},
\end{aligned}
$$

which implies $B\left(S_{\alpha}(t)\right)$ is a multifractal process [32]. So, it is a useful tool to characterize asset returns feature.

In summary, the stochastic differential equation (3) presents many important financial properties, such as selfsimilarity, leptokurtic feature, and long memory. So, we assert that model equation (3) characterizes the price of underlying well. In Section 3, we investigate the option pricing problem under the assumption that the underlying of the option contract is driven by the time changed Brownian motion.

\section{Option Pricing under the Subordinated Models}

Let $V(S, t)$ be the value at time $t$ of an European option on the above underlying with expiration data $T$ and exercise price $K$ and the boundary condition

$$
V(S, T)= \begin{cases}\left(S_{T}-K\right)^{+}, & \text {call option, } \\ \left(K-S_{t}\right)^{+}, & \text {put option. }\end{cases}
$$

Since $S_{\alpha}(t)$ is independent of $B(\tau)$, then the subordinated geometric Brownian motion (4) can be seen as a geometric Brownian motion with stochastic volatility

$$
d S_{t}=\mu S_{t} d t+\widetilde{\sigma}(t) S_{t} d B(t)
$$

where $\widetilde{\sigma}(t)$ is a stochastic process driven by $S_{\alpha}(t)$. The wellknown option pricing problem with stochastic volatility can be found in [33]. Let $\eta=\mathrm{d} S_{\alpha}(t) / \mathrm{d} t$, then $\widetilde{\sigma}(t)=\sigma \eta^{1 / 2}$. The relation can also be given as

$$
\int_{0}^{t} \tilde{\sigma}^{2}(s) \mathrm{d} s=\int_{0}^{t} \sigma^{2} \mathrm{~d} S_{\alpha}(t)
$$

where $S_{\alpha}(t)$ is the increasing process, so the stochastic integral with respect to $S_{\alpha}(t)$ can be defined as Riemann integral. And, $S_{\alpha}(t)$ is independent of $B(t)$, and then $\widetilde{\sigma}(t)$ is also independent of $B(t)$. By using Itô theory, equation (3) yields

$$
S_{t}=S_{0} \exp \left\{\int_{0}^{t}\left(\mu-\frac{\tilde{\sigma}^{2}(s)}{2}\right) \mathrm{d} s+\int_{0}^{t} \tilde{\sigma}(s) \mathrm{d} B(s)\right\} .
$$

We can get the log distribution of $S_{t}$ which is no longer the normal distribution, but yields to

$$
\begin{aligned}
P(X(t), t)= & \int_{0}^{t} \frac{1}{\sqrt{2 \pi \sigma^{2}} \tau} \exp \\
& \cdot\left(-\frac{\left(X-\left(\mu t-\sigma^{2} \tau / 2\right)\right)^{2}}{2 \sigma^{2} \tau}\right) g(\tau, t) \mathrm{d} \tau,
\end{aligned}
$$

where $g(\tau, t)$ is the probability density function of $S_{\alpha}(T)$, given as $1 / \tau H_{1}^{1}{ }_{1}^{0}\left[\tau /\left.t^{\alpha}\right|_{(1,1)} ^{(1, \alpha)}\right]$ (see [34]). By using the properties of $S_{\alpha}(t)$, we also can get the mean

$$
E\left[S_{t}\right]=S_{0} e^{\mu t}
$$

and the second moment

$$
E\left[S_{t}^{2}\right]=S_{0}^{2} e^{2 \mu t} \int_{0}^{+\infty} e^{\sigma^{2} \tau} g(\tau, t) \mathrm{d} \tau=S_{0}^{2} e^{2 \mu t} E_{\alpha}\left(\sigma^{2} t^{\alpha}\right) .
$$

Then, we can get the variance of $S_{t}$, given as

$$
\operatorname{Var}\left(S_{t}\right)=E\left[S_{t}^{2}\right]-E\left[S_{t}\right]^{2}=S_{0}^{2} e^{2 \mu t}\left(E_{\alpha}\left(\sigma^{2} t^{\alpha}\right)-1\right)
$$

The results reduce to the ones showed in [35] as $\alpha \longrightarrow 1$. The second moment does not exist when the stock price is driven by Levy process [36].

Here, we cannot build a portfolio to hedge the two stochastic terms $\tilde{\sigma}(t)$ and $B(t)$. So, the self-financing method used in the classical Black-Sholes model is not suitable for this model, and under hypothesis (4), the market is incomplete. 
Now, let $X_{t}=S_{t} e^{-r t}$, where $r$ is the risk-free rate, so $X_{t}$ denotes the discounted value of the risk assets, then

$$
d X_{t}=(\mu-r) X_{t} d t+\widetilde{\sigma}(t) X_{t} d B(t)
$$

Let

$$
B^{Q}(t)=\frac{(\mu-r)) t}{\widetilde{\sigma}(t)}+B(t)
$$

Then, equation (16) yields

$$
d X_{t}=\tilde{\sigma}(t) X_{t} d B^{Q}(t)
$$

Here, let $Q$ be the probability measure defined as

$$
Q(A)=\int_{A} Z_{t} \mathrm{~d} P
$$

where $A \in \mathscr{F}$ and

$$
Z_{t}=\exp \left\{-\frac{1}{2} \int_{0}^{t}\left(\frac{\mu-r}{\tilde{\sigma}(s)}\right)^{2} \mathrm{~d} s-\int_{0}^{t} \frac{\mu-r}{\tilde{\sigma}(s)} \mathrm{d} B(s)\right\} .
$$

By Girsanov theory [37], we know $B^{Q}(t)$ is Brownian motion under the probability measure $Q$. So, the discounted value $X_{t}$ is the $\mathscr{F}_{S_{\alpha}(t)}$-martingale with respect to $Q$. Here, $\mathscr{F}_{S_{\alpha}(t)}$ denotes the information about the history of asset prices $S_{t}$ up to time $S_{\alpha}(t)$, dependent on the stochastic process $S_{\alpha}(t) . \mathscr{F}_{S_{\alpha}(t)}$ is also called filtration and is interpreted as the background information which is available for the investor. The more time it proceeds, the more the information is revealed to the investor. Then, the price of the European option is given by

$$
V(S, t)=E^{Q}\left[e^{-r(T-t)} V(S, T) \mid \mathscr{F}_{S_{\alpha}(t)}\right],
$$

where $V(S, T)$ is given by equation (8), and under the new measure $Q$, the asset price yields

$$
S_{t}=S_{0} \exp \left\{\int_{0}^{t}\left(r-\frac{\tilde{\sigma}^{2}(s)}{2}\right) \mathrm{d} s+\int_{0}^{t} \tilde{\sigma}(s) \mathrm{d} B^{Q}(s)\right\} .
$$

On the one hand, according to the option pricing formula (21), we know that the option price $V(S, t)$ depends on the information filtration $\mathscr{F}_{S_{\alpha}(t)}$, which is random, so is the option price $V(S, t)$. They are both dependent on stochastic term $S_{\alpha}(t)$. In fact, in the real market, each people get his price of option based on the obtained information. The information people received are different, which leads them to price their options differently, which is consistent with the option pricing formula (21). So, the option price is not unique, which implies the market is incomplete. On the other hand, the stochastic volatility $\tilde{\sigma}(t)$ leads to the equivalent martingale is not unique, so the arbitrage opportunity exists and we cannot get the unique price of option under our assumption. All these results are caused by the randomness of $S_{\alpha}(t)$.

Form the option pricing formula (21), we know the option price cannot be easily calculated, which is dependent on the catched information $\mathscr{F}_{S_{\alpha}(t)}$, and the randomness of $S_{\alpha}(t)$ leads to the nonuniqueness of option price. However, for $t=0, S_{\alpha}(t)$ is no longer stochastic, but fixed, $S_{\alpha}(0)=0$. At $t=0$, the catched information is zero for everyone, and they are equal in the market. So, the option price is unique, and the market is complete at that moment. We should notice that the martingale measure $Q$ has one important advantage. When $\alpha \longrightarrow 1, S_{\alpha}(t)$ reduces to $t$ and $Q$ reduces to the martingale measure of the classical BS model, which is known to be arbitrage-free and complete [8]. Thus, in the following, we concentrate on the martingale measure $Q$ and deduce the analytical option pricing formula.

When $t=0$, the option pricing formula (21) yields to

$$
V(S, t=0)=E^{Q}\left[e^{-r T} V(S, T)\right] .
$$

Here, we consider the European option, Call; then,

$$
\begin{aligned}
V(S, t=0)= & E^{Q}\left[e^{-r T}\left(S_{T}-K\right) \mathbb{1}_{S_{T}>K}\right], \\
= & \int_{0}^{\infty} E^{Q}\left[e^{-r T}\left(S_{0} e^{r T-\sigma^{2} \tau / 2+\sigma B^{Q}(\tau)}-K\right) \mathbb{1}_{S_{0} e^{r T-\sigma^{2} \tau / 2+\sigma B}(\tau)>K}\right] \\
& g(\tau, T) \mathrm{d} \tau,
\end{aligned}
$$

where 1 is the indicator function. Here,

$$
\begin{aligned}
& E^{Q}\left[\left(e^{-r T} S_{0} e^{r T-\sigma^{2} \tau / 2+\sigma B^{Q}(\tau)}\right) 1_{S_{0} e^{r T-\sigma^{2} \tau / 2+\sigma B}(\tau)>K}\right] \\
& =E^{Q}\left[\left(e^{-r T} S_{0} e^{r T-\sigma^{2} \tau / 2+\sigma B^{Q}(\tau)}\right) 1_{B(\tau)>\ln \left(\mathrm{K} / S_{0}\right)-r T+\sigma^{2} \tau / 2 / \sigma}\right] \\
& =S_{0} \int_{h}^{+\infty} e^{-\sigma^{2} \tau / 2+\sigma \sqrt{\tau} x} \frac{1}{\sqrt{2 \pi}} e^{-x^{2} / 2} \mathrm{~d} x, \\
& \cdot\left(h=\frac{\ln \left(K / S_{0}\right)-r T+\sigma^{2} \tau / 2}{\sigma \sqrt{\tau}}\right) \\
& =S_{0} N\left(d_{1}\right)
\end{aligned}
$$

where $N(\cdot)$ is the cumulative function of normal distribution and $d_{1}=\ln \left(S_{0} / K\right)+r T+\sigma^{2} \tau / 2 / \sigma \sqrt{\tau}$. We also can get

$$
E^{Q}\left[e^{-r T} K \mathbb{1}_{S_{0} e^{r T-\sigma^{2} \tau / 2+\sigma B}(\tau)}>K\right]=e^{-r T} \mathrm{KN}\left(d_{2}\right),
$$

where $d_{2}=d_{1}-\sigma \sqrt{\tau}$. So, we have

$$
V(S, t=0)=S_{0} N_{\alpha}\left(d_{1}\right)-K e^{-r T} N_{\alpha}\left(d_{2}\right) .
$$

Here, $N_{\alpha}(\cdot)=\int_{0}^{+\infty} N(\cdot) g(\tau, T) \mathrm{d} \tau$. We should notice that $g(\tau, T)=\delta(\tau-T)$ as $\alpha \longrightarrow 1$; then, the option pricing formula equation (27) reduces to the classical Black-Sholes model $[1,2]$. The results are consistent with those obtained in $[15,38]$ when the order of space fractional derivative reduces to 2 .

The model tells us the option price is fair (or unique) when it is firstly written. The reason lies in the information about this option obtained by the straddle both sides are zero at that moment. After that, different people will get different information about this option from market, which leads to the different price. Then, trading between different people happens. The more information obtained, the more accurate the price is. From the definition of $S_{\alpha}(t)$, we know $S_{\alpha}(t) \leq t$, 


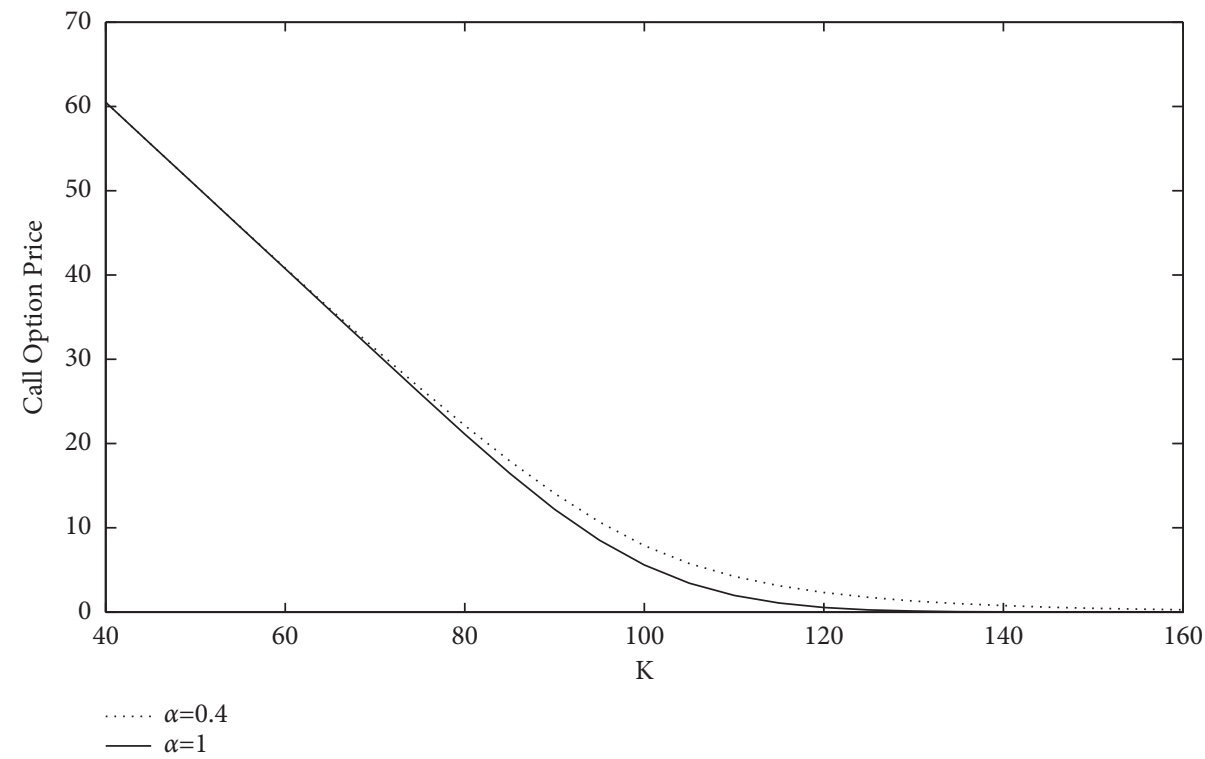

Figure 2: The Call option price with different $\alpha$. Here, $r=0.05, \sigma=0.25, S_{t}=100$, and $T=0.25$. The Monte Carlo method is used for stochastic simulation, and the result is obtained by simulating 50,000 times.

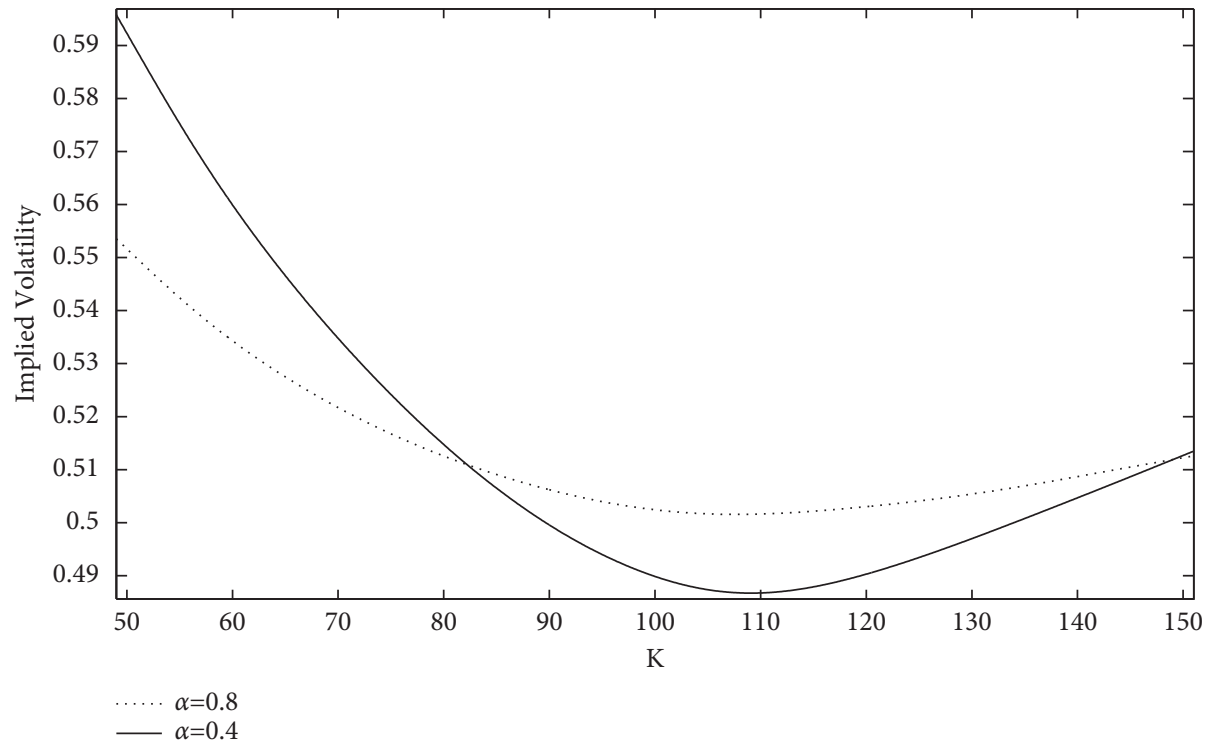

FIGURE 3: The implied volatility smile with different $\alpha$.

for $\alpha>0$, then $\mathscr{F}_{S_{\alpha}(t)} \in \mathscr{F}_{t}$, which indicates people can only capture part of the information that matches the real market. For $\alpha=1$, each person can capture all the information about the option, which implies this is a perfect market. So, the investors will get the same price for the option, there is no arbitrage in the market. But, in real world, different people set the different reasonable price of option based on the information they captured, which makes options market actively traded.

By comparing this obtained formula with the classical Black-Sholes pricing formula in Figure 2, we find that the call option price increases with the decrease of $\alpha$. In the real world, the option is an insurance product, which is used to hedge the risk of the underlying assets. As the return of the underlying assets is supposed to be heavy tailed, the risk is redistributed, which results in an adjustment of the option price. The risk increases with the decrease of the parameter $\alpha$ which leads to the higher option price, especially the strike price $K$ is near the expectation of $S_{T}$. This behavior can be observed in situations when we face some kind of unexpected or sudden change of regime, such as a black day on the market, the bankruptcy of a company trading on the market, and a natural disaster [39]. Such sudden change also can be modeled by considering GBM with resetting [40, 41]. In short time, people are willing to spend more money to protect their assets.

As we know, if the Black-Scholes model is completely correct, then the implied volatility should be constant. In 


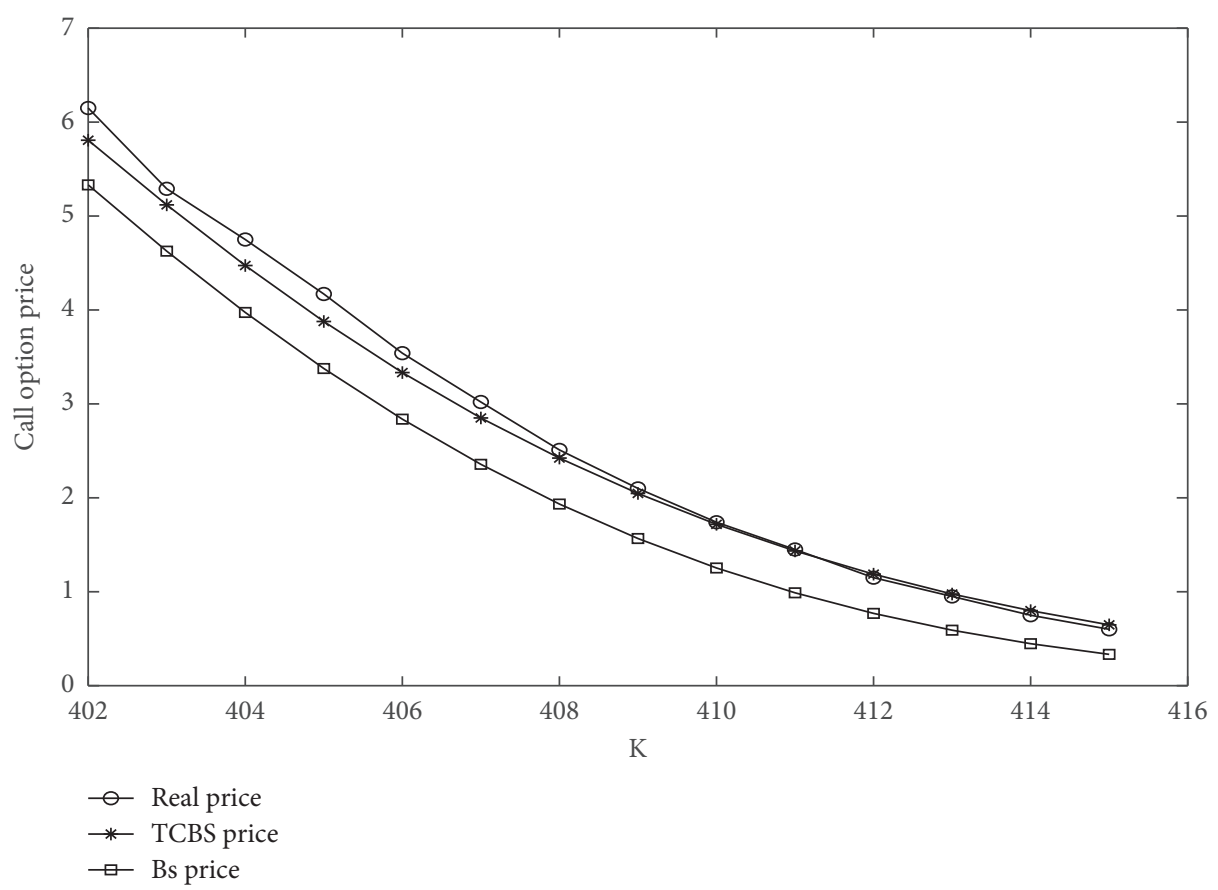

FIGURE 4: Comparison between the real Call option price with the one obtained by different models under different strike price K. Here, BS price stands for the price obtained by classical Black-Scholes model, and TCBS stands for the price obtained by equation (27) with timechanged Brownian motion.

reality, it is widely recognized that the implied volatility curve resembles a "smile," meaning it is a convex curve of the strike price. This empirical phenomenon called "volatility smile" in option markets [42]. The implied volatility can be obtained by two steps: step one, use option pricing formula (27) to get the option price. Step two, use the obtained option price to derive the volatility by the classical Black-Sholes formula. By following the above two steps, we obtain the implied volatility smile with different $\alpha$. From Figure 3, we find the smaller the parameter $\alpha$ is, the larger the curvature is. The volatility tends to be horizontal as $\alpha \longrightarrow 1$. Moreover, following the same procedure, we can deduce that the option price will be lower than the classical one if the parameters $\alpha>1$, and the volatility will be concave, which is called "volatility cry."

We choose SPDR S\&P500 ETF call option (SPY) as the empirical research object. The quotation data is April 7, 2021, and the expiration date is April 18, 2021. The index of S\&P500 ETF is 406.12 (source: https://yahoo.com). Here, we imply the rescaled range analysis method $(\mathrm{R} / \mathrm{S}$ analysis method) to estimate the parameter $\alpha$, which yields $\alpha=0.94$. In Figure 4, we find the proposed option price is higher than the classical Black-Scholes price and closer to the real price.

\section{Conclusions}

In this paper, we employ a time changed Brownian motion, whose PDF is proved to follow the fractional diffusion equation. The process exhibits many financial characters, such as self-similarity, leptokurtic, and long memory features. Then, we apply this model to the option pricing problem. The underlying of the option contract is supposed to be driven by a subordinated geometric Brownian motion. Under this assumption, we obtain the evaluation formula of an European option. In contrast with the classical model, higher option price and "volatility smile" are found in our model. These phenomenon is consistent with the real market. An empirical analysis is also made to show the accuracy of our model. Recently, the empirical results indicate the rough stochastic volatility model can capture the real market well. How will the option price be if the rough stochastic volatility is driven by $B\left(S_{\alpha}(t)\right)$, which is interesting research contents. Study on the time averaged MSD and nonergodicity of this subordinated geometric Brownian motion is also meaningful [40, 43, 44]. Finally, we expect that the results obtained here may be useful to the discussion of the anomalous diffusion systems and complex financial market $[45,46]$.

\section{Data Availability}

The data used to support the findings of this study are included within the article.

\section{Conflicts of Interest}

The authors declare that no conflicts of interest exist regarding this manuscript.

\section{Acknowledgments}

This work was supported by the Subject of Philosophy and Social Science Planning in Zhejiang Province (no. 21NDJC168YB) and National Natural Science Foundation of China (no. 11801288). 


\section{References}

[1] F. Black and M. Scholes, "The pricing of options and corporate liabilities," Economy, vol. 81, no. 3, pp. 637-654, 1973.

[2] R. C. Merton, "Theory of rational option pricing," Bell Journal of Economics and Management Science, vol. 4, no. 1, pp. 141-183, 1973.

[3] G. Jumarie, "Stock exchange fractional dynamics defined as fractional exponential growth driven by (usual) Gaussian white noise. Application to fractional Black-Scholes equations," Insurance: Mathematics and Economics, vol. 42, no. 1, pp. 271-287, 2008.

[4] S. G. Kou, "A jump-diffusion model for option pricing," Management Science, vol. 48, no. 8, pp. 1086-1101, 2002.

[5] H. E. Leland, "Option pricing and replication with transactions costs," The Journal of Finance, vol. 40, no. 5, pp. 1283-1301, 1985.

[6] P. P. Boyle and T. Vorst, "Option replication in discrete time with transaction costs," The Journal of Finance, vol. 47, no. 1, pp. 271-293, 1992.

[7] M. Mastinsek, "Discrete-time delta hedging and the BlackScholes model with transaction costs," Mathematical Methods of Operations Research, vol. 64, pp. 227-236, 2006.

[8] M. Magdziarz, "Black-Scholes formula in subdiffusive regime," Journal of Statistical Physics, vol. 136, no. 3, pp. 553-564, 2009.

[9] M. Magdziarz, S. Orzeł, and A. Weron, "Option pricing in subdiffusive Bachelier model," Journal of Statistical Physics, vol. 145, no. 1, pp. 187-203, 2011.

[10] M. Magdziarz and J. Gajda, "Anomalous dynamics of BlackScholes model time-changed by inverse subordinators," Acta Physica Polonica B, vol. 43, no. 5, pp. 1093-1110, 2012.

[11] C. N. Angstmann, B. I. Henry, and A. V. McGann, “Timefractional geometric Brownian motion from continuous time random walks," Physica A: Statistical Mechanics and its Applications, vol. 526, Article ID 121002, 2019.

[12] G. Krzyzanowski, M. Magdziarz, and L. Plociniczak, "A weighted finite difference method for subdiffusive BlackCScholes model," Computers and Mathematics with Applications, vol. 80, pp. 653-670, 2020.

[13] V. Stojkoski, T. Sandev, L. Basnarkov, L. Kocarev, and R. Metzler, "Generalised geometric Brownian motion: theory and applications to option pricing," Entropy, vol. 22, no. 12, p. 1432, 2020.

[14] L. Lv, J. Xiao, L. Fan, and F. Ren, "Correlated continuous time random walk and option pricing," Physica A: Statistical Mechanics and its Applications, vol. 447, pp. 100-107, 2016.

[15] J.-P. Aguilar, C. Coste, and J. Korbel, "Series representation of the pricing formula for the European option driven by spacetime fractional diffusion," Fractional Calculus and Applied Analysis, vol. 21, no. 4, pp. 981-1004, 2018.

[16] J.-P. Aguilar and J. Korbel, "Option pricing models driven by the space-time fractional diffusion: series representation and applications," Fractal and Fractional, vol. 2, no. 1, p. 15, 2018.

[17] A. Dahlbokum, "Empirical performance of option pricing models based on time-changed Lévy processes," 2010.

[18] S. Klingler, Y. S. Kim, and S. T. Rachev, "Option pricing with time-changed Lévy processes," Applied Financial Economics, vol. 23, no. 15, pp. 1231-1238, 2013.

[19] Z. Tong, "Option pricing in stochastic volatility models driven by fractional Lévy processes," International Journal of Financial Markets and Derivatives, vol. 5, pp. 56-75, 2016.
[20] R. N. Mantegna and H. E. Stanley, "Scaling behaviour in the dynamics of an economic index," Nature, vol. 376, no. 6535, pp. 46-49, 1995.

[21] M. Magdziarz, A. Weron, and K. Weron, "Fractional FokkerPlanck dynamics: stochastic representation and computer simulation," Physical Review E, Statistical, Nonlinear, and Soft Matter Physics, vol. 75, Article ID 016708, 2007.

[22] R. Metzler and J. Klafter, "The random walk's guide to anomalous diffusion: a fractional dynamics approach," Physics Reports, vol. 339, no. 1, pp. 1-77, 2000.

[23] Z. Guo, "Option pricing under the Merton model of the short rate in subdiffusive Brownian motion regime," Journal of Statistical Computation and Simulation, vol. 87, no. 3, pp. 519-529, 2017.

[24] L. Lv, J. Xiao, L. Zhang, and L. Gao, "Solutions for a generalized fractional anomalous diffusion equation," Journal of Computational and Applied Mathematics, vol. 225, no. 1, pp. 301-308, 2009.

[25] L. Lv, W. Qiu, and F. Ren, "Fractional Fokker-Planck equation with space and time dependent drift and diffusion," Journal of Statistical Physics, vol. 149, no. 4, pp. 619-628, 2012.

[26] G. Premaratne and A. Bera, "A test for symmetry with leptokurtic financial data," Journal of Financial Econometrics, vol. 3, no. 2, pp. 169-187, 2005.

[27] E. Scalas, R. Gorenflo, and F. Mainardi, "Fractional calculus and continuous-time Finance," Physica A: Statistical Mechanics and its Applications, vol. 284, no. 1-4, pp. 376-384, 2000.

[28] F. Mainardi, M. Raberto, R. Gorenflo, and E. Scalas, "Fractional calculus and continuous-time finance II: the waitingtime distribution," Physica A: Statistical Mechanics and its Applications, vol. 287, no. 3-4, pp. 468-481, 2000.

[29] M. Raberto, E. Scalas, and F. Mainardi, "Waiting-times and returns in high-frequency financial data: an empirical study," Physica A: Statistical Mechanics and its Applications, vol. 314, no. 1-4, pp. 749-755, 2002.

[30] E. Scalas, "The application of continuous-time random walks in finance and economics," Physica A: Statistical Mechanics and its Applications, vol. 362, no. 2, pp. 225-239, 2006.

[31] R. T. Baillie, "Long memory processes and fractional integration in econometrics," Journal of Econometrics, vol. 73, no. 1, pp. 5-59, 1996.

[32] R. Cont, "Empirical properties of asset returns: stylized facts and statistical issues," Quantitative Finance, vol. 1, no. 2, pp. 223-236, 2001.

[33] S. L. Heston, "A closed-form solution for options with stochastic volatility with applications to bond and currency options," Review of Financial Studies, vol. 6, no. 2, pp. 327-343, 1993.

[34] M. M. Meerschaert, D. A. Benson, H. P. Scheffler, and B. Baeumer, "Stochastic solution of space-time fractional diffusion equations," Physical Review. E, Statistical, Nonlinear, and Soft Matter Physics, vol. 65, Article ID 041103, 2002.

[35] A. G. Cherstvy, D. Vinod, E. Aghion, I. M. Sokolov, and R. Metzler, "Scaled geometric Brownian motion features subor superexponential ensemble-averaged, but linear time-averaged mean-squared displacements," Physical Review, vol. 103, no. 6, Article ID 062127, 2021.

[36] J. Y. Li, H. S. Shu, and X. Kan, "European option pricing with transaction costs in Levy jump environment," Abstract and Applied Analysis, vol. 2014, Article ID 513496, 2014.

[37] J. C. Cox, J. E. Ingersoll, and S. A. Ross, "An intertemporal general equilibrium model of asset prices," Econometrica, vol. 53, no. 2, pp. 363-384, 1985. 
[38] J. Korbel and Y. Luchko, "Modeling of financial processes with a space-time fractional diffusion equation of varying order," Fractional Calculus and Applied Analysis, vol. 19, no. 6, pp. 1414-1433, 2016.

[39] H. Kleinert and J. Korbel, "Option pricing beyond BlackScholes based on double-fractional diffusion," Physica A: Statistical Mechanics and its Applications, vol. 449, pp. 200214, 2016.

[40] V. Stojkoski, T. Sandev, L. Kocarev, and A. Pal, "Geometric Brownian motion under stochastic resetting: a stationary yet nonergodic process," Physical Review, vol. 104, no. 1, Article ID 014121, 2021.

[41] V. Stojkoski, P. Jolakoski, A. Pal, T. Sandev, K. Ljupco, and M. Ralf, "Income inequality and mobility in geometric Brownian motion with stochastic resetting: theoretical results and empirical evidence of non-ergodicity," 2021, https://arxiv. org/abs/2109.01822.

[42] E. Derman, I. Kani, and N. Chriss, "Implied trinomial tress of the volatility smile," Journal of Derivatives, vol. 3, no. 4, pp. 7-22, 1996.

[43] O. Peters and W. Klein, "Ergodicity breaking in geometric Brownian motion," Physical Review Letters, vol. 110, no. 10, Article ID 100603, 2013.

[44] A. G. Cherstvy, D. Vinod, and E. Aghion, "Time averaging, ageing and delay analysis of financial time series," New Journal of Physics, vol. 19, no. 6, Article ID 063045, 2017.

[45] Y. Ren, R. liang, Y. Qiu, and B. Xiao, "Answer to an open problem proposed by R Metzler and J Klafter," Journal of Physics A: Mathematical and General, vol. 39, no. 18, pp. 4911-4919, 2006.

[46] M. Parkinson, "The extreme value method for estimating the variance of the rate of return," Journal of Business, vol. 53, no. 1, pp. 61-65, 1980. 